\title{
A Special Summer Pruning Method Significantly Increases Fruit Weight, Ascorbic Acid, and Dry Matter of Kiwifruit ('Jinyan', Actinidia eriantha $\times$ A. chinensis)
}

Guang-Lian Liao and Xiao-Biao Xu

College of Forestry, Jiangxi Agricultural University, Jiangxi Provincial Key Laboratory of Silviculture, Nanchang, Jiangxi, P.R. China, 330045; and College of Agronomy, Jiangxi Agricultural University, Kiwifruit Institute of Jiangxi Agricultural University, Nanchang, Jiangxi, P.R. China, 330045

Qing Liu, Min Zhong, Chun-Hui Huang, Dong-Feng Jia, and Xue-Yan Qu

College of Agronomy, Jiangxi Agricultural University, Kiwifruit Institute of Jiangxi Agricultural University, Nanchang, Jiangxi, P.R. China, 330045

Additional index words. flavor, flesh color, fruit quality, kiwifruit, shoot twisting and squeezing, taste

Abstract. Jinyan (Actinidia eriantha $\times$ A. chinensis) is one of the gold-fleshed kiwifruit cultivars currently being promoted in south China. However, its fruit dry matter is usually less than $16 \%$, which seriously affects fruit quality including taste and flavor. This causes a financial loss to growers: not only are the prices paid for the fruit low because of their bad reputation for quality, but some orchards have been removed. Improvement of fruit quality is essential. In this study, a method is described for squeezing and twisting flowering shoots before flowering and removing the distal vegetative parts of flowering shoots after fruit set. The effects on fruit quality were determined. The dry matter of fruit was increased by $6.6 \%$. Fruit size also increased as did the chlorophyll a content and the chlorophyll:carotenoid ratio. The significantly increased fruit dry matter, resulting in significant increases in fruit soluble solids concentrations $(P<0.01)$, thereby possibly improving fruit taste. Fruit weight, fruit length, and carotenoid and ascorbic acid concentrations were significantly enhanced in comparison with controls $(P<0.01)$, increasing by $20 \%, 7 \%, 12 \%$, and $19 \%$, respectively. However, there was no significant difference in soluble sugar concentrations, titratable acid concentrations, and the reduced chlorophyll b concentrations. This research provides a practical method to increase fruit dry matter, and hence a way to allow fruit quality to reach commercial requirements for cultivars such as Jinyan, which under previous management systems had significant shortcomings in fruit flavor and taste.

'Jinyan', a kiwifruit cultivar selected and promoted by the Wuhan Botanical Garden, Chinese Academy of Sciences during the last decade, is one the most promising goldfleshed cultivars for the south of China. It is thought to be an interspecific hybrid from the cross Actinidia eriantha as mother, with $A$. chinensis as father (Zhong et al., 2015). Many reports have shown that 'Jinyan' produces good crops of fruit, with attractive, goldcolored flesh. It is true that in vitro experiments by Wang et al. (2019) showed 'Jinyan' to be highly sensitive to kiwifruit canker disease, a bacterial disease that is fatal and destroys commercial kiwifruit orchards. But our own field trials in Gannan, Jiangxi and reports from Pujiang, Sichuan (W.B. Zhao, personal communication) indicate that 'Jinyan' is more tolerant of the disease than other cultivars widely planted in south China, such as 'Hongyang', a red-fleshed kiwifruit cultivar. In Pujiang County, Sichuan, 4000 ha of
Jinyan have been planted, making it the most widespread gold-fleshed kiwifruit cultivar there. Considerable plantings have also been established in Jiangxi, Zhejiang, Hunan, and other provinces in the south of China.

However, the dry matter of the mature fruit from Fengxin County, Jiangxi is generally less than $16 \%$, and this seriously affects fruit taste and flavor. Dry matter is an important index of fruit quality; it determines the fruit flavor and taste of Actinidia fruit when they are eating ripe (Burdon et al., 2004; Nardozza et al., 2011; Wu et al., 2013). Low dry matter eventually becomes a financial issue for kiwifruit growers, and the problem seriously affects the economics of kiwifruit orchards because of the dislike of customers of such inferior fruit (Jaeger et al., 2011; Wu, 2019).

Although there are many ways to improve dry matter (Patterson and Currie, 2019; Wu et al., 2012), there are only a few reports on how the method of pruning affects fruit quality (Miller et al., 2001; Patterson and Currie, 2011; Thorp et al., 2018). In addition, fertilization, exogenous hormones, moderate drought stress, and other methods can be used to improve the dry matter of fruit crops (Sale and Lyford, 1990; Wu, 2015; Wu et al., 2012). However, fruit growers prefer to use pruning, girdling, summer pruning, and so forth to control shoot growth and vegetative growth to achieve high fruit dry matter, fruit size, and desired crop load (Patterson and Currie, 2019; Wu, 2019), because such methods are safer, without potential chemical contamination, more effective, and have fewer adverse effects on both the vine and the environment.

In this study, a new summer canopy management methodology has been developed, in which the terminal parts of flowering shoots are twisted and squeezed before flowering, then removed after fruit set. Aspects of fruit quality such as flesh color, flavor, and taste of the fruit from treated shoots were evaluated and compared with controls. The aim was to provide a practical method to increase fruit dry matter, thereby allowing fruit quality to reach commercial requirements.

\section{Materials and Methods}

\section{Plant materials}

The orchards used belonged to the Institute of Kiwifruit, Jiangxi Agricultural University, and were they were located in Fengxin County, Jiangxi Province, China. 'Jinyan' was the predominant fruiting kiwifruit cultivar planted in the orchard. The male cultivar Moshan 4 was used as the pollinizer planted at a ratio of 1 male to 8 females along the row. The orchard was under standard management. Summer pruning method was applied in late April, on sunny days when the local temperature was 25 to $28^{\circ} \mathrm{C}$.

A modified commercial cultivation system was used in this study with a support structure (Fig. 1A). In the present study, six healthy vines of nearly the same growth status were selected for the experiment. They were randomly divided into three groups (replicates) with two vines in each group: one treated, the other the control. Five flowering shoots were selected in each vine. At flower popcorn stage, they were twisted and squeezed by hand, distal to the top flower bud (Fig. 1B and C); untwisted fruit shoots were used as controls (Fig. 1D). Twisting was carried out by putting one hand at the position of the top flower bud and slowly rotating the other hand in a clockwise direction and squeezing the distal part of the shoot. Not all flowering shoots were treated - only vigorously growing, nonterminating flowering shoots.

Determination of size, weight, and inner quality of fruit

About 60 fruit from different directions (east, south, west, and north) of the twisted and squeezed shoots of each vine were harvested when their soluble solids concentrations reached $7.5 \%$ (Chen et al., 2019). These were placed at room temperature 
$\left(25{ }^{\circ} \mathrm{C}, 95 \% \mathrm{RH}\right)$. Thirty fruit randomly selected from each treatment were weighed, then measured to calculate fruit shape index (length/width) and the dry matter. After 2 weeks of storage at room temperature when the fruit had become edible (firmness was $1 \mathrm{~kg} / \mathrm{cm}^{2}$ ) (Liao et al., 2019a), fruit quality was evaluated by measuring dry matter, flesh color, and soluble solids concentration following the methods described (Chen et al., 2019; Liao et al., 2019a; Seal et al., 2013; Wu et al., 2013). Soluble solids concentrations were measured by a digital refractometer $(0 \%$ to $50 \%$ "pocket" PAL-1; Atago) and flesh color by Chromameter CR-400 (Konica Minolta, Japan; the illuminant of the chromameter was D65). The concentrations of soluble sugars, titratable acids, pigment, and ascorbic acid concentrations were measured after the fruit pulp was ground in liquid nitrogen (Liao et al., 2019b).

\section{Data analysis}

The experimental data were statistically analyzed and processed with Microsoft Excel 2016 and SPSS 17.0. A two-tailed Student's $t$ test was used to detect differences at $P \leq 0.05$ and $P \leq 0.01$.

\section{Results}

Effects of twisting the shoots on the fruit size, flesh color, and weight

Twisting and squeezing the shoots significantly affected fruit flesh color (Fig. 2); the hue angle of treated fruit and control fruit was $91.67 \pm 1.36^{\circ}$ and $98.12 \pm 3.65^{\circ}$, respectively. The closer the hue angle is to $90^{\circ}$, the more golden the flesh. Fruit weight and length were significantly increased $(P<0.01)$ by, respectively, $20 \%$ and $7 \%$. With this treatment, the yield increased by $15 \%$ compared with the

Received for publication 18 May 2020. Accepted for publication 9 Aug. 2020.

Published online 15 September 2020

This work was supported by the National Natural Science Foundation of China (Grant No. 31760559 , 31760567), key research and development plan of Jiangxi Science and Technology Department (Grant No. 20192ACB60002). We have no competing interests.

We thank Jinhu $\mathrm{Wu}$ and Ross Ferguson of the New Zealand Institute for Plant \& Food Research Ltd. for their constructive comments and suggestions, and for revision of the English.

G.-L.L. is a PhD candidate and working in fruit germplasm resources and biotechnology.

G.-L.L. and X.-B.X. conceived and designed the experiment, analyzed and interpreted the data, and prepared the manuscript. Q.L., M.Z., C.-H.H., D.-F.J., and X.-Y.Q. participated in fruit sample collection, also with internal qualities and external qualities analysis. All authors read and approved the final manuscript. All data included in this study are available upon request by contact with the corresponding author.

X.-B.X. is the corresponding author. E-mail: xbxu@ jxau.edu.cn.

This is an open access article distributed under the CC BY-NC-ND license (https://creativecommons. org/licenses/by-nc-nd/4.0/).
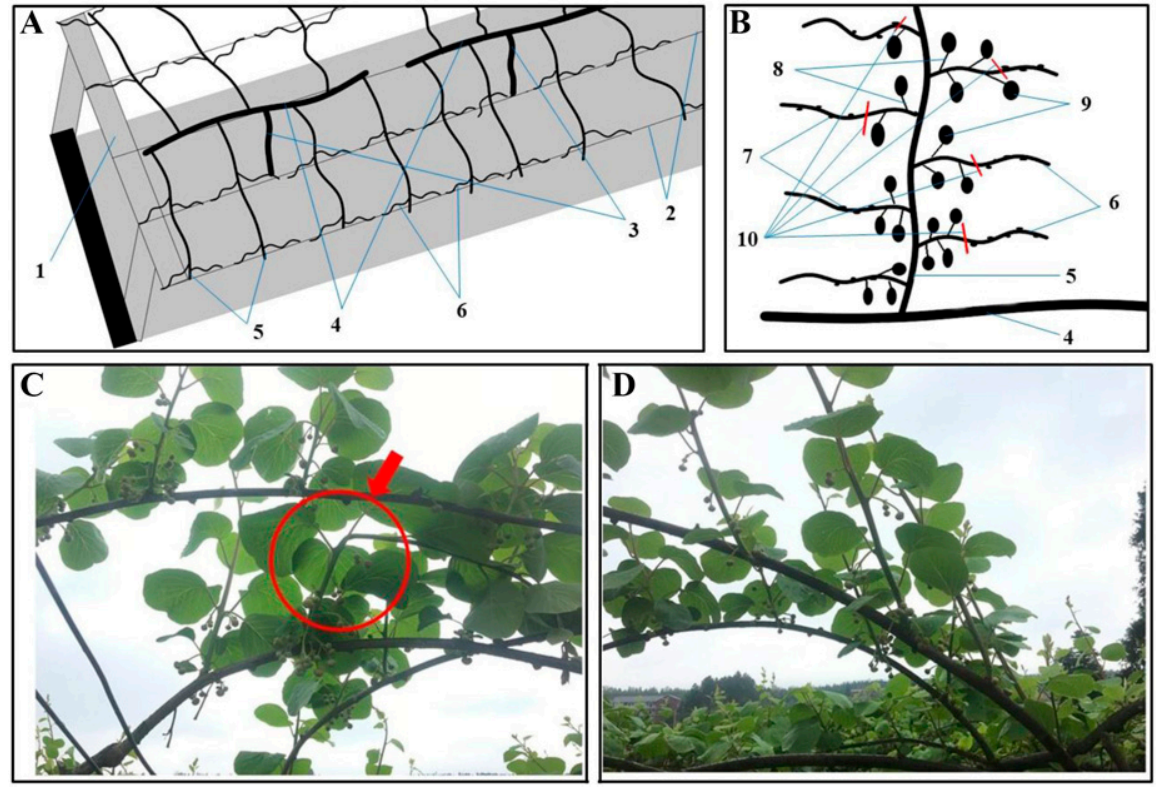

Fig. 1. Sketch maps and photos separately show the modified supporting commercial kiwifruit planting system used in this study with the procedure and position of this summer horticultural pruning technique. (A) Supporting commercial kiwifruit planting system with the main kiwifruit canopy structure. (B) The fruiting shoots produced on one-year-old canes; the red line marks the twisted and squeezed position of the shoots (last flower position), (C) The red circle shows the real twisted and squeezed shoot with flower buds before bloom. (D) Untreated shoots with flower buds as controls. The arable numbers in (A) and (B) separately show main supporting structure with a beam (1) and iron wires (2), and vine's structure with trunks (3), main leaders (4), one-year-old wood canes (5), flowering shoots (later fruiting shoots) (6), nonterminating flowering shoots (Ferguson, 1990) (7), flower or fruit stalks (peduncle) (8), flower buds or fruitlets (9), and the position where the twisting and squeezing of flowering shoots is applied (10).

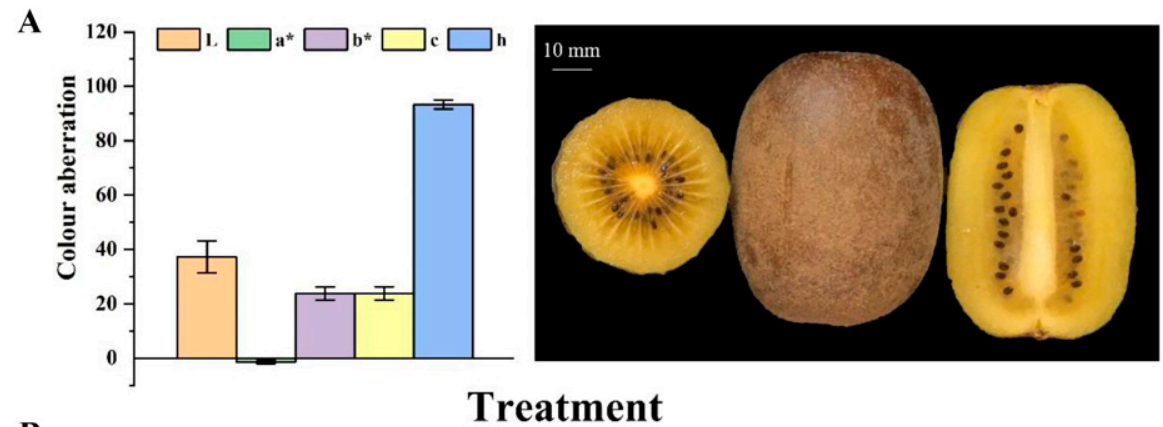

B

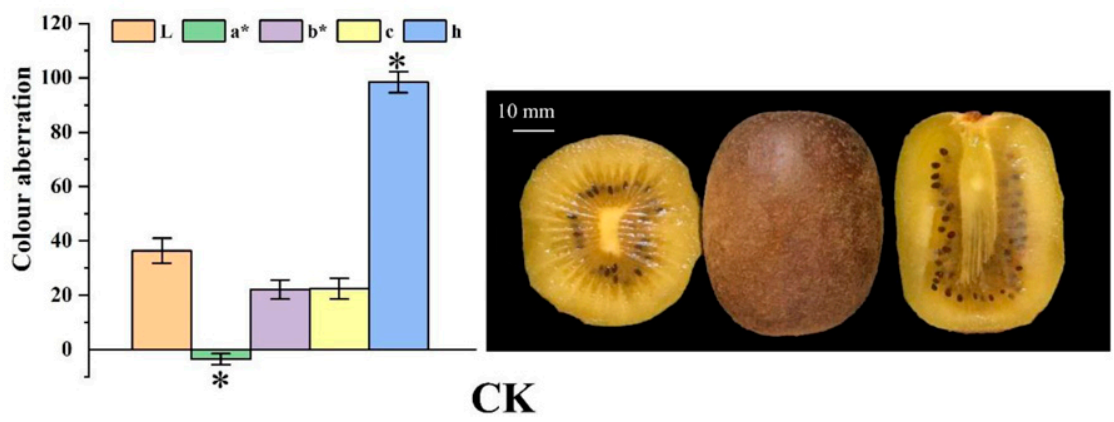

Fig. 2. (A) Fruit attributes (size, shape, and flesh color) of 'Jinyan' (Actinidia eriantha $\times$ A. chinensis) from the treatment with twisted and squeezed fruit shoots before bloom, to compare with (B) untreated shoots (CK). "L" stands for brightness: the higher the value, the brighter it will be. The " $\mathrm{a}^{*}$ " value represents the red/ green color difference index: the larger the positive value is, the deeper the red is; and the smaller the negative value is, the deeper the green is. The " $b^{*}$ " value represents the yellow/blue color difference index: the larger the positive value is, the deeper the yellow is; and the smaller the negative value is, the deeper the blue is. The "c" stands for chroma (the degree of saturation or purity). The " $\mathrm{h}$ " is the hue angle: when the value of hue angles is closer to $90^{\circ}$, the flesh is more golden. The * shows a significant difference found at $P \leq 0.05$ on the same traits tested between treatment and control CK. 
control group. The fruit shape index did not change significantly (Fig. 3).

Effects on fruit internal qualities of twisting and squeezing the shoots before flowering

Soluble solids concentrations, dry matter, titratable acid, and soluble sugar concentrations. The summer pruning method significantly increased fruit dry matter $(P<0.05)$ by $0.84 \%$ units ( $15.25 \%$ to $16.09 \%)$ and soluble solids concentrations $(P<0.01)$ by $0.43 \%$ units (from $16.57 \%$ to $17 \%$ ). There was no significant effect on titratable acid and soluble sugar concentrations (Fig. 4).

Ascorbic acid, chlorophyll a, chlorophyll $b$, and carotenoid concentrations. The summer pruning method significantly increased ascorbic acid $(P<0.01)$, chlorophyll a $(P<$ $0.05)$, and carotenoid concentrations $(P<$ 0.01 ) by $19.35 \%, 12.45 \%$, and $12.43 \%$, respectively (Fig. 5). Chlorophyll b was significantly lower, $18.23 \%$, than in the control $(P<0.05)$

Total soluble solids:acidity ratio, sugar:acid ratio, and chlorophyll:carotenoid ratio. The
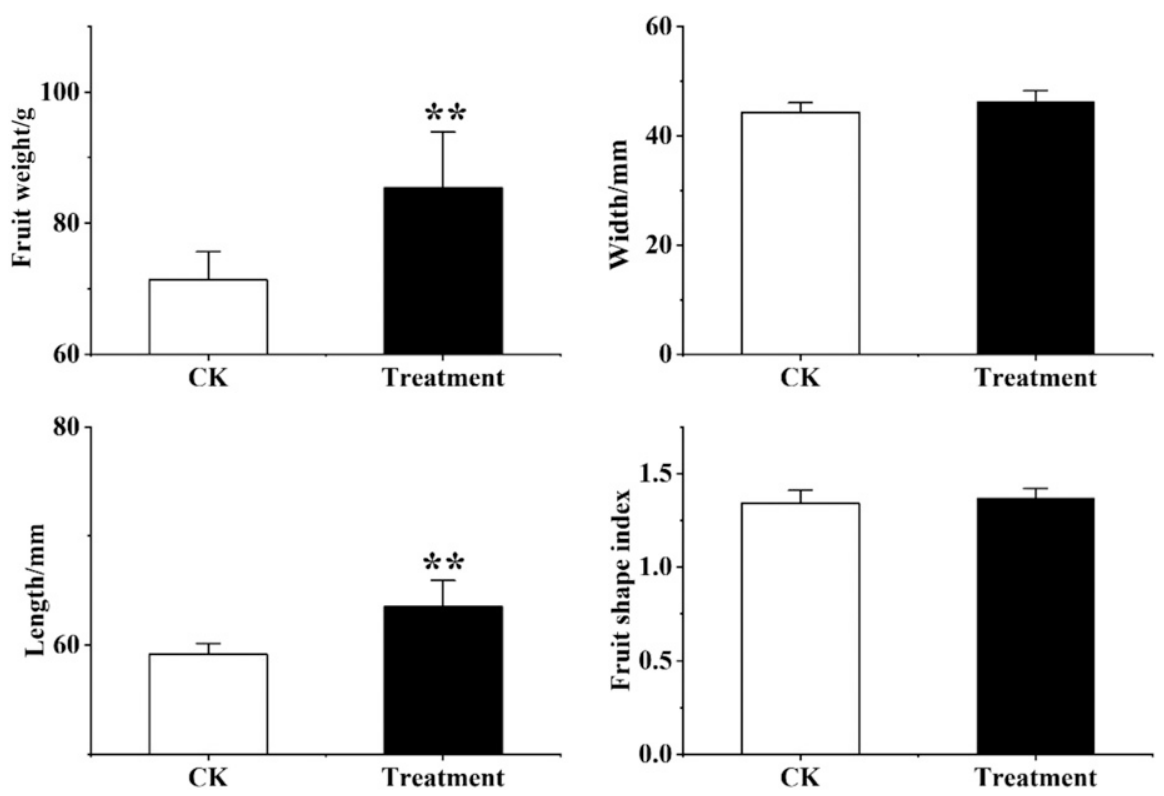

Fig. 3. Effects of twisted and squeezed shoots before bloom on fruit and weight of 'Jinyan' (Actinidia eriantha $\times A$. chinensis) fruit (** shows a significant difference found between treatment and control $\mathrm{CK}$ at $P \leq 0.01)$.
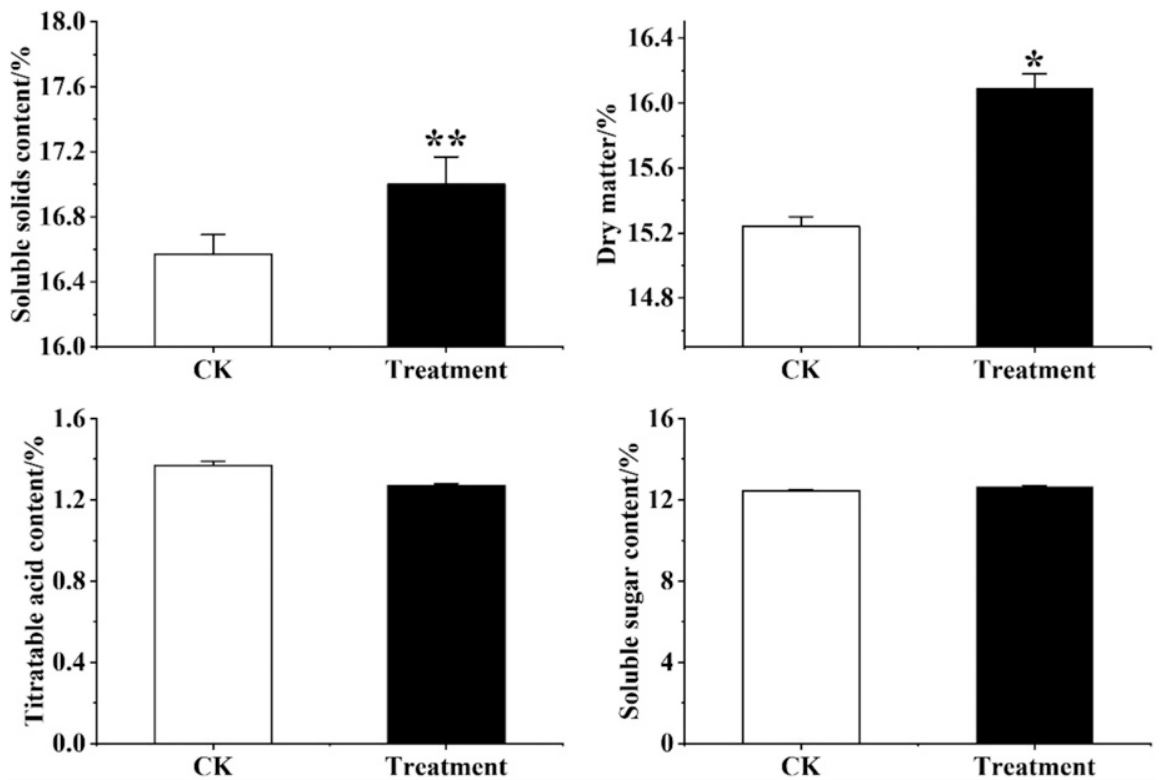

Fig. 4. Effects of the twisted and squeezed shoots before bloom on soluble solids concentrations, dry matter, titratable acid, and soluble sugar concentrations of 'Jinyan' (Actinidia eriantha $\times$ A. chinensis) fruit (* and ** show a significant difference found between treatment and control $\mathrm{CK}$ either at $P \leq 0.05$ or $P \leq 0.01)$. taste and flavor of the fruit depend mainly on the total soluble solids:acids ratio and the sugar:acid ratio when the total soluble solids content does not vary. The color of the fruit flesh depends on the chlorophyll:carotenoid ratio. The treatment increased the total soluble solids:acids ratio $(P<0.01)$, while the chlorophyll:carotenoid ratio had been reduced $(P<0.01)$ by the treatment. However, no significant change was found on the sugar:acid ratio (Fig. 6).

\section{Discussion}

The economic value of kiwifruit is determined by fruit weight, crop yield, and fruit quality - mainly fruit size, flesh taste, and flavor. Dry matter is especially important in kiwifruit as an index of fruit taste and flavor quality (Nardozza et al., 2011; Wu et al., 2012, 2013). Many factors affect dry matter accumulation and distribution, such as temperature, humidity, fruit load, and sunny days during the fruit growing season, as all these factors affect photosynthesis and carbohydrate product accumulation (Adams and Holder, 1992). We can improve dry matter accumulation by management techniques such as pruning, soil management, and measures affecting vine growth and fruit development, if we understand the physiological mechanisms involved. Management by pruning does not involve the use of chemicals, and there is therefore no risk of chemical residues. The pruning methods developed by us result in greater dry matter accumulation and do not harm the vines. There is a vigorous period of vegetative growth before flowering. During this period, carbohydrate or photosynthate could supply shoot growth, but the flower buds are also developing and need nutrients (Chao and Li, 2000). So, we infer that the new summer pruning method improves the fruit dry matter by reducing the sink strength of the growing vegetative shoots. At this stage, vegetative shoots without flower buds or fruit should be removed and the fruiting shoots are twisted and squeezed, resulting in increased supplies of the products from photosynthesis to flower buds. However, not all the shoots should be treated in this way; the short and spur shoots and the shoots used to renew vines should not be twisted, because this could reduce overall canopy photosynthetic capacity and lead to inadequate carbohydrate supplies to the developing flower buds (Albert et al., 2010). After the long shoots are twisted and squeezed, the leaves can still continue to photosynthesize and supply photosynthate for flower development and fruitlet setting and growth. While the leaves' and shoots' own growth is reduced after damage, they do not compete so strongly for photosynthate. This may be an important reason why the new summer pruning method improves the dry matter, fruit weight, and other economic fruit traits. Zero-leaf pruning as developed by Patterson et al. (2009) removes growing tips from shoots where there is sufficient leaf area present and where the shoot is not required to 

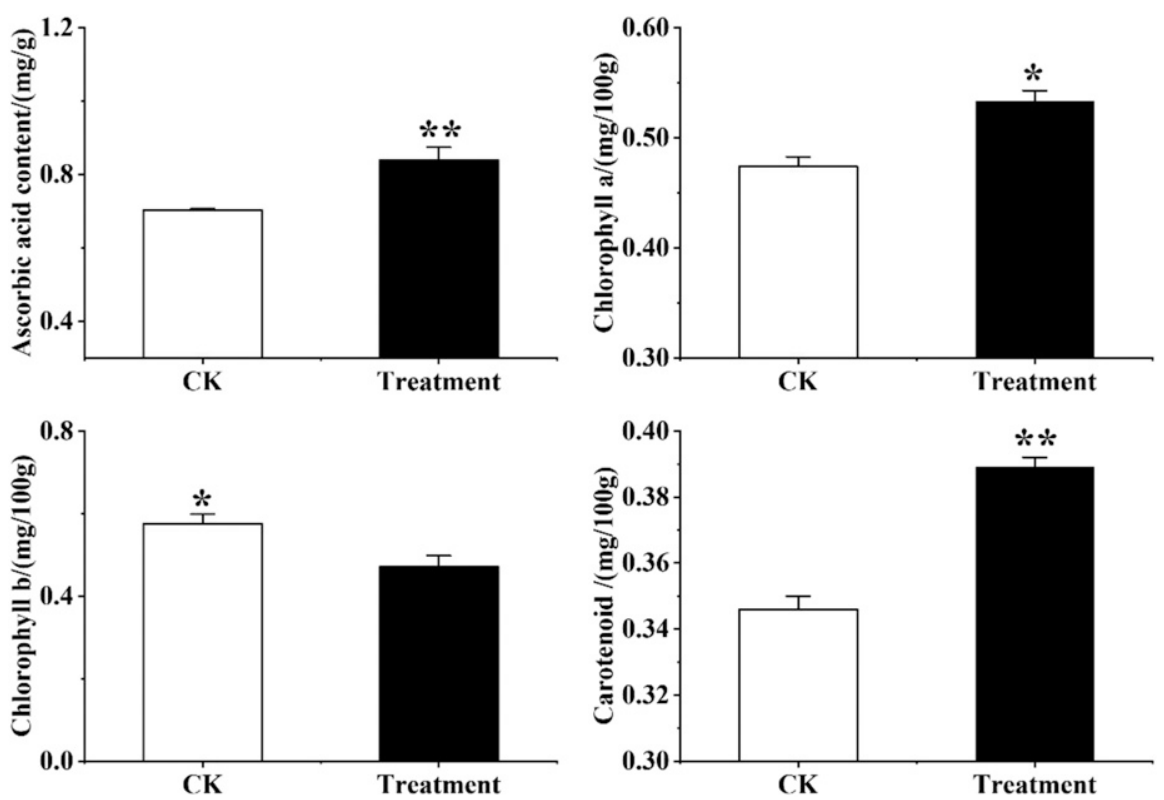

Fig. 5. Effects of the twisted and squeezed shoots on ascorbic acid, chlorophyll a, chlorophyll b, and carotenoid concentrations of 'Jinyan' (Actinidia eriantha $\times$ A. chinensis) fruit (* and ** show a significant difference found between treatment and control $\mathrm{CK}$ either at $P \leq 0.05$ or $P \leq 0.01$ ).
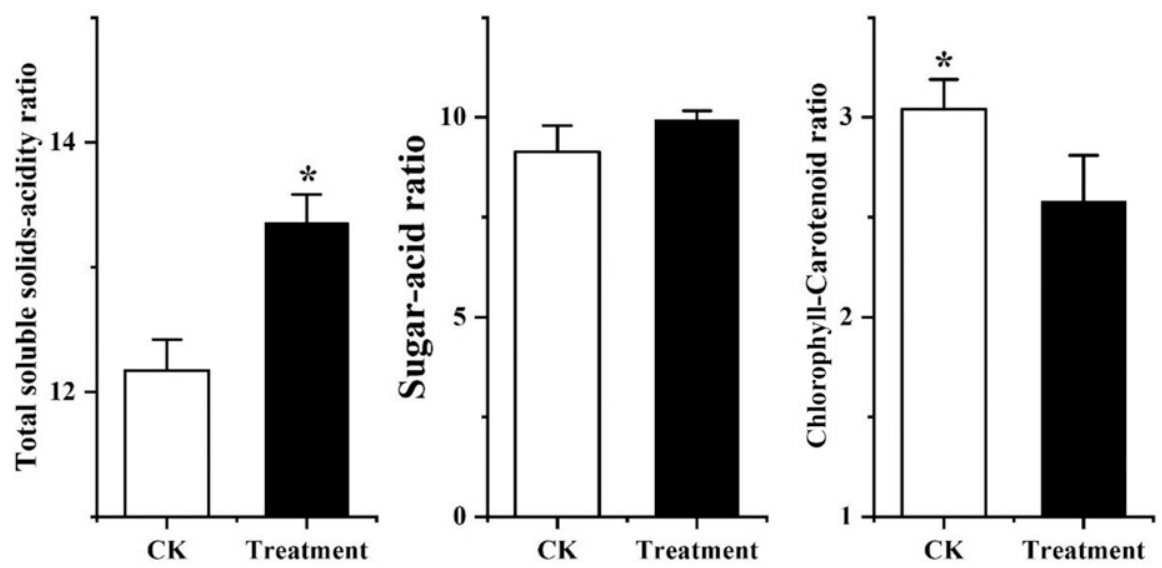

Fig. 6. Effects of the twisted and squeezed shoots on total soluble solids-acidity, sugar:acid ratio and chlorophyll:carotenoid ratio of 'Jinyan' (Actinidia eriantha $\times$ A. chinensis) fruit $(*$ shows a significant difference between treatment and control CK found at $P \leq 0.05$ ).

be part of the following year's fruiting canopy. The pruning method developed in our study is rather different, because zero leaf pruning removes all vegetative growth distal to the last fruit after fruit set. Zero leaf pruning was originally intended to maintain an open canopy. Our method starts earlier, before flowering, to ensure an adequate supply of photosynthate to the developing flower, and our method is then followed by zero leaf pruning.

The quality of flower buds and fruitlet development has a large effect on the ultimate fruit quality. The new summer pruning method described here is aimed at improving the quality of flowers and fruitlet development, so as to increase the dry matter and weight of the fruit. This has been achieved in tomatoes (Xiao et al., 2005) and pears (Liao et al., 2003). In addition, many studies have shown that dry matter is positively correlated with soluble solid concentrations (Liao et al., 2019 c). But how this new summer twist method alters the accumulation of pigments is unclear, and further research is needed. The summer pruning method increased soluble solids concentrations, but it had no effect on soluble sugar or titratable acid concentrations. This is consistent with studies on grapes (Jia, 2014). The soluble solids concentration is made up of soluble sugars, acids, and other soluble compounds present in fruit juice. Although both soluble solids concentration and soluble sugar were higher than in the control, only soluble solids concentration showed a significant difference. It is likely that the new summer pruning method has no significant effect on the components and contents of organic acids (Jiang et al., 2020). The difference in the ratio of total soluble solids:acids leads to the difference in taste. In general, chlorophyll makes the flesh appear green, and carotenoids make the flesh appear golden. In this study, the new summer pruning method increased the carotenoid concentrations and reduced chlorophyll concentrations, resulting in the fruit flesh appearing more golden (Huang et al., 2014). This was also further confirmed by the hue angle value.

\section{Conclusion}

Our results showed that the dry matter of kiwifruit can be effectively increased by twisting and squeezing the distal ends of flowering shoots before bloom and then removing them after fruit set. This new summer pruning method not only significantly improves dry matter but also increases the chlorophyll a and the chlorophyll:carotenoid ratio $(P<0.05)$. In addition, the soluble solids concentrations, fruit weight, fruit length, carotenoid and ascorbic acid concentrations also significantly increase $(P<0.01)$. However, no significant differences have been found in soluble sugar concentrations, titratable acid concentrations, and reduced chlorophyll $b$ concentrations. These changes eventually result in the flesh becoming more golden and increasing the dry matter of fruit. The method described increases the fruit dry matter, allowing the quality point of more fruit to reach commercial requirements.

\section{Literature Cited}

Adams, P. and R. Holder. 1992. Effects of humidity, $\mathrm{Ca}$ and salinity on the accumulation of dry matter and $\mathrm{Ca}$ by the leaves and fruit of tomato (Lycopersicon esculentum). J. Hort. Sci. 67(1): 137-142.

Albert, T., K. Karp, M. Starast, and T. Paal. 2010. The effect of mulching and pruning on the vegetative growth and yield of the half-high blueberry. Agron. Res. 8(1):759-768.

Burdon, J., D. McLeod, N. Lallu, J. Gamble, M. Petley, and A. Gunson. 2004. Consumer evaluation of 'Hayward' kiwifruit of different atharvest dry matter contents. Postharvest Biol. Technol. 34:245-255.

Chao, W.J. and X. Li. 2000. Effect of extra-short pruning on the flower bud formation of grapevine. China Fruits (4):20-22.

Chen, L., S.Y. Wang, M. Zhong, C.H. Huang, G.L. Liao, and X.B. Xu. 2019. Effects of pollens from the 10 selected Actinidia male genotypes on 4 commercial planting kiwifruit female cultivars in Southern China. N. Z. J. Crop Hort. Sci. 47(3):155-169.

Ferguson, A.R. 1990. Stem, branches, leaves and roots of the kiwifruit vine, p. 58-70. In: I.J. Warrington and G.C. Weston (eds.). Kiwifruit: Science and management. Ray Richards Publisher, Auckland.

Huang, C.H., J. Gao, X.H. Zhang, C.J. Chen, X.Y. $\mathrm{Qu}$, and X.B. Xu. 2014. Study on the changes of contents of pigments of yellow-fleshed kiwifruit during fruit development. J. Food Sci. 31(4):617-623.

Jaeger, S.R., R. Harker, C.M. Triggs, A. Guson, R.L. Campbell, R. Jackman, and C. RequejoJackman. 2011. Determining consumer purchase intentions: The importance of dry matter, size and price of kiwifruit. J. Food Sci. 76: S177-S184. 
Jia, Y. 2014. Research on the standardized technology of pruning grape floral clusters. Nanjing Agricultural University, Master's thesis. <http:// cdmd.cnki.com.cn/Article/CDMD-103071016039897.htm>.

Jiang, Z.Q., Q. Huang, D.F. Jia, M. Zhong, J.J. Tao, G.L. Liao, C.H. Huang, and X.B. Xu. 2020. Characterization of organic acid metabolism and expression of related genes during fruit development of Actinidia eriantha 'Ganmi 6'. Plants 9:332.

Liao, G.L., X.B. Xu, M. Zhong, C.H. Huang, G.Q Tu, B.M. Li, J.J. Tao, X.Y. Qu, S.G. Zhao, and J.H. Leng. 2019a. A novel mid-maturing cultivar with high dry matter content from seedlings of 'Jinfeng' kiwifruit (Actinidia chinensis). Eur. J. Hort. Sci. 84(5):294-301.

Liao, G.L., Y.Q. He, X.S. Li, M. Zhong, C.H. Huang, S.Y. Yi, Q. Liu, and X.B. Xu. 2019 b. Effects of bagging on fruit flavor quality and related gene expression of AsA synthesis in Actinidia eriantha. Scientia Hort. (Amsterdam) 256:108511.

Liao, G.L., Z.Y. Li, C.H. Huang, M. Zhong, J.J. Tao, X.Y. Qu, L. Chen, and X.B. Xu. 2019c. Genetic diversity of inner quality and SSR association analysis of wild kiwifruit (Actinidia eriantha). Scientia Hort. (Amsterdam) 248:241247.

Liao, L.A., Z.G. Li, and J.M. Cao. 2003. Introduction experiment of 'Cuiguan' pear and effect of trimming the branches on its economic properties. J. Central South For. Univ. 23(2):79-81.
Miller, S.A., F.D. Broom, T.G. Thorp, and A.M. Barnett. 2001. Effects of leader pruning on vine architecture, productivity and fruit quality in kiwifruit (Actinidia deliciosa cv. Hayward). Scientia Hort. (Amsterdam) 91(3):189-199.

Nardozza, S., G. Joanna, A. Lauren, W. Mark, J. Michael, J.Q. Feng, and F.R. Harker. 2011. Dry matter content and fruit size affect flavor and texture of novel Actinidia deliciosa genotypes. J. Sci. Food Agr. 91(4):742-748.

Patterson, K., P. Blattmann, and M. Currie. 2009. Zero-leaf pruning, getting the balance right. Kiwifruit 196:20-22.

Patterson, K.J. and M.B. Currie. 2011. Optimizing kiwifruit vine performance for high productivity and superior fruit taste. Acta Hort. 913:257-268.

Patterson, K.J. and M.B. Currie. 2019. Optimsing kiwifruit productivity in New Zealand. Scr. Hort. 20:81-86.

Sale, P.R. and P.B. Lyford. 1990. Cultural, management and harvesting practices for kiwifruit in New Zealand, p. 247-296. In: I.J. Warrington and G.C. Weston (eds.). Kiwifruit: Science and management. Ray Richards Publisher, Auckland.

Seal, A.G., J.K. Dunn, H.N. De Silva, T.K. McGhie, and R.C.M. Lunken. 2013. Choice of pollen parent affects red flesh color in seedlings of diploid Actinidia chinensis (kiwifruit). N. Z. J. Crop Hort. Sci. 41(4):207-218.

Thorp, T.G., A.M. Barnett, M. Blattmann, D. Hedderley, and R. Mayston. 2018. Optimising vine management to increase yields and improve quality of Zespri SunGold kiwifruit
(Actinidia chinensis var. chinensis 'Zesy002'). Acta Hort. 1218:397-404.

Wang, F., J. Li, K. Ye, P. Liu, H. Gong, Q. Jiang, B. Qi, and Q. Mo. 2019. An in vitro Actinidia bioassay to evaluate the resistance to Pseudomonas syringae pv. actinidiae. Plant Pathol. J. $35: 372-380$.

Wu, J.H. 2015. Pollen effects on fruit attributes and seed properties in colchicine-induced autotetraploids of red-fleshed kiwifruit (Actinidia chinensis Planch.). J. Hort. Sci. Biotechnol. 90:695-703.

Wu, J.H. 2019. Cultivar, environment and integration of horticultural practices will determine the future of the kiwifruit industry. Scr. Hort. 20:171-178.

Wu, J.H., A.R. Ferguson, B.G. Murray, A.M. Duff, Y. Jia, C. Cheng, and P.J. Martin. 2013. Fruit quality in induced polyploids of Actinidia chinensis. HortScience 48:701-707.

Wu, J.H., A.R. Ferguson, G.B. Murray, Y. Jia, P.M. Datson, and J. Zhang. 2012. Induced polyploidy dramatically increases the size and alters the shape of fruit in Actinidia chinensis. Ann. Bot. (Lond.) 109:169-179.

Xiao, S.G., P.H. Zhou, E. Heuvelink, and Z.M. Liu. 2005. Production and distribution of tomato dry matter in greenhouse based on library regulation. Chinese J. Tropical Crops. (4):24-27.

Zhong, C.H., P. Zhang, F. Han, and D.W. Li. 2015. Studies on characterization of fruit development of interspecific hybrid cultivar-'Jinyan'. J. Food Sci. 32(6):1152-1160. 